\title{
Simulation of mechanical misalignments in a cone- beam micro-CT system
}

\author{
I. Vidal-Migallón, Student Member, IEEE, M. Abella, A. Sisniega, Student Member, IEEE, J.J. Vaquero, Senior \\ Member, IEEE, M. Desco
}

\begin{abstract}
X-ray CT images usually show artefacts due not only to physical effects -e.g., beam hardening-, but also to misalignments that remain after mechanical calibration. These artefacts become particularly noticeable in the case of high spatial resolution systems and in hybrid systems, such as PETCT, SPECT-CT scanners, which rely on a correct registration of emission and CT data. Hence, slight mechanical misalignments affect the quality of the $C T$ images and any attenuation correction methods or further quantification based on them. We implemented a computer simulator of these artefacts on a conebeam, flat-panel based micro-CT scanner. Using this simulator, we studied the effect of these different misalignments (pitch and roll tilts, skew and shifts) on reconstructed images.
\end{abstract}

\section{INTRODUCTION}

$\mathrm{X}^{-1}$ -RAY CT images usually show artefacts due not only to physical effects -e.g., beam hardening-, but to mechanical misalignments that remain after calibration as well. These artefacts become particularly harmful in the case of high spatial resolution systems and in hybrid systems, such as PETCT, SPECT-CT scanners, which rely on a correct registration of emission and CT data to obtain useful anatomical information. Hence, slight mechanical misalignments affect the quality of the CT images and any attenuation correction methods or further quantification based on it.

Several studies have discussed the effects of certain shifts and rotations in the rotation axis [1] or in the source and detector panel [2]. Sun et al. described the effects of sizeable misalignments and proposed an FBP algorithm with embedded misalignment correction [3]. This paper studies the effect of several detector panel misalignments on $\mathrm{CT}$ images reconstructed with a variation of Feldkamp's filtered backprojection (FBP) algorithm and the tolerance to these misalignments of a cone-beam micro-CT system.

\section{MATERIAL AND METHODS}

The misalignment simulator is entirely programmed using IDL 6.4 and emulates the activity of a cone-beam micro-CT system for small-animal imaging [4] in two ways: it can add the effects of mechanical misalignments on pre-existing 2D projections obtained from the micro-CT scanner or it can

Manuscript received November 14,2008 . This work is partially funded by the CD-TEAM Project, CENIT Program, Spanish Ministerio de Industria, and with grants from the Ministerio de Educación y Ciencia, projects TEC200764731 and TEC2008-06715-C02-01.

I. Vidal-Migallón, M. Abella, A. Sisniega, J. J. Vaquero and M. Desco are with the Unidad de Medicina y Cirugía Experimental, Hospital General Universitario Gregorio Marañón (e-mail: desco@mce.hggm.es). produce a set of projections around synthetic phantoms in $1^{\circ}$ steps, based on parameters that describe the real system, e.g. source to detector distance. In an attempt to isolate the effects of each type of misalignment, measurements will be shown on synthetic phantom images. These misalignments are: tilts (pitch and roll of the detector panel), skew (i.e., skew [2] or rotation around the central ray), X-shift and Y-shift of the detector panel with respect to the source.
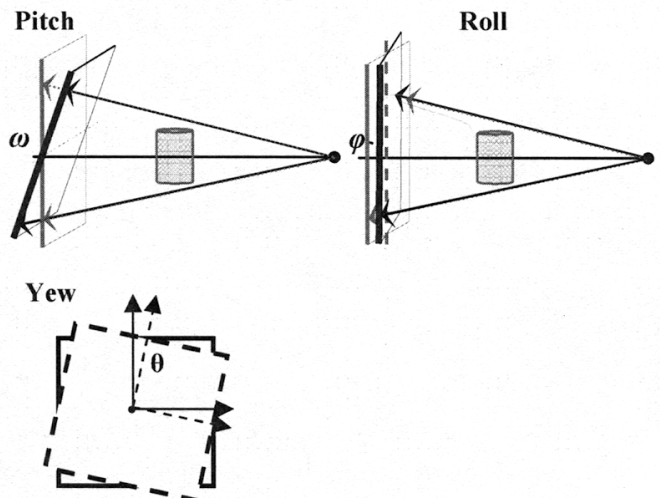

Fig. 1: Different angles of misalignment.

Setting the origin of coordinates in the virtual detector, the projection algorithm determines for each $2 \mathrm{D}$ projection pixel, the path of the photon ray from the source to the pixel in the detector and calculates the contribution of points in that path to the total attenuation value registered in the pixel. Tilt misalignments (i.e., pitch and roll of the detector panel) are embedded in the projection algorithm, while the effects of skew and shifts are incorporated into the resulting projections.

Different synthetic phantoms of sizes similar to that of small animals have been created with characteristics that would help visualise artefacts easily. Pixel size for these results is $0.25 \mathrm{~mm}$.

\section{A. Tilt}

Let $\mathrm{A}$ be the point of impact of a certain ray in a detector panel without any tilt misalignment and $A^{\prime}$ the point in which the same ray impacts when the panel has an angle of tilt, $\boldsymbol{\omega}$. Pitch and roll of the detector panel affects the location of the pixels hit by the rays, but not the actual path of the ray.

Therefore, our simulation calculates the impact point on a correctly aligned panel and the contributions of the points in 
the path of the corresponding ray; it then maps that value to the point in the tilted detector panel.

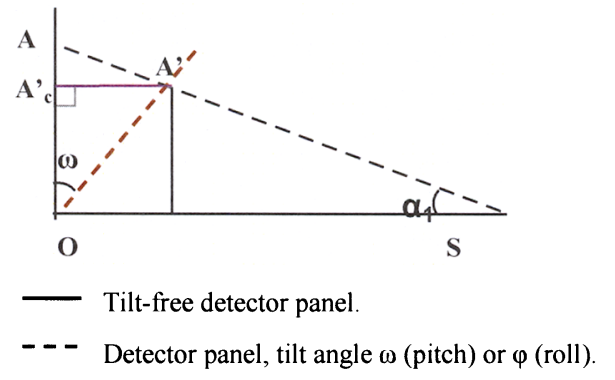

Fig. 2: Impact points of a ray on a tilted panel (A') and correctly aligned panel (A).

\section{B. Skew, $X$-shift and $Y$-shift}

An angle of rotation $\theta$ of the detector panel around the central ray produces a constant misalignment for all points in the projection and every angle around the object. Similarly, vertical and horizontal shifts in the detector panel have no effect on the shape of the projection and require that we shift the stack of 2D projections a certain number of pixels in either direction.

\section{RESULTS}

\section{A. Roll tilt}

Images affected by roll tilt showed deformation and blurring. The diameter of the spheres shown in the direction of maximum distortion was measured on the same slice: $\emptyset_{\text {onginal }}=$ $4.07 \mathrm{~mm}, \emptyset_{8^{\circ}}=4.25 \mathrm{~mm}, \emptyset_{20^{\circ}}=4.46 \mathrm{~mm}$.

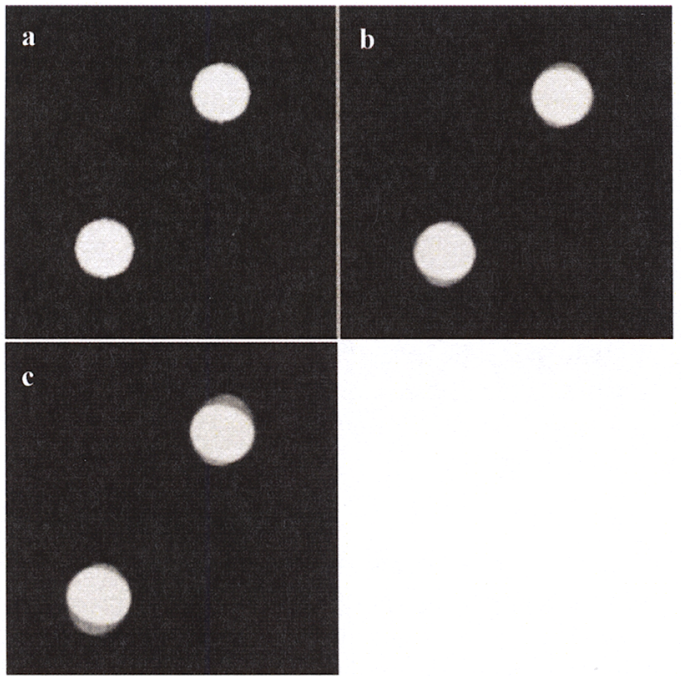

Fig. 3: Comparison between images obtained from an aligned detector (a), an $8^{\circ}$-roll (b) and a $20^{\circ}$-roll (c). Image dimensions: $200 \times 200 \times 70$ pixels. Axial view.

\section{B. Pitch tilt}

Pitch misalignment has been found to produce a deformation dependent on the degree of tilt. Thus: $\emptyset_{\text {original }}=$ $4.08 \mathrm{~mm}, \varnothing_{8^{\circ}}=4.26 \mathrm{~mm}, \varnothing_{15^{\circ}}=4.47 \mathrm{~mm}$. The effect of misalignments under $5^{\circ}$ produced a deformation that increased the sphere's diameter in less than $3 \%$ and was not visible
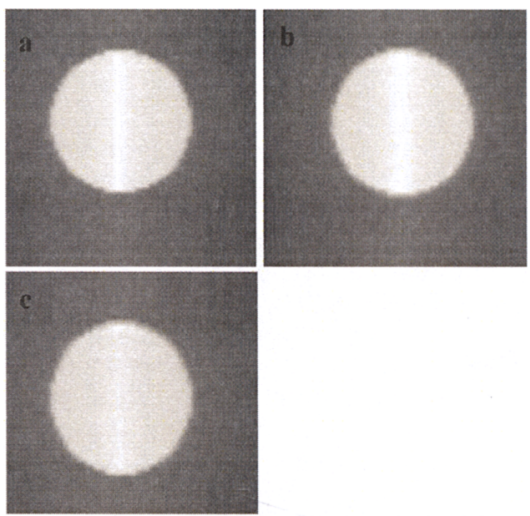

Fig. 4 : Comparison between details from images obtained from an aligned detector (a), an $8^{\circ}$-roll (b) and a $20^{\circ}$-roll. Axial view. Image dimensions: $100 \times 100 \times 240$

\section{Skew (rotation)}

$1^{\circ}$ of rotation around the central ray produced double edges visible in the coronal and sagittal views of a simple cylindrical phantom of dimensions: $280 \times 280 \times 100$.


Fig. 5: $1^{\circ}$ (a) and $8^{\circ}($ b) skew around the central ray. Image dimensions: $280 \times 280 \times 100$ pixels. Coronal view.

\section{D. $X$-shift and $Y$-shift}

Fig. 6 shows the effect of 1 pixel (at this resolution, $0.25 \mathrm{~mm}$ ) of X-shift and a 6-pixel Y-shift. Y-shift seems to only affect the sharp slice after a Feldkamp FBP reconstruction. 


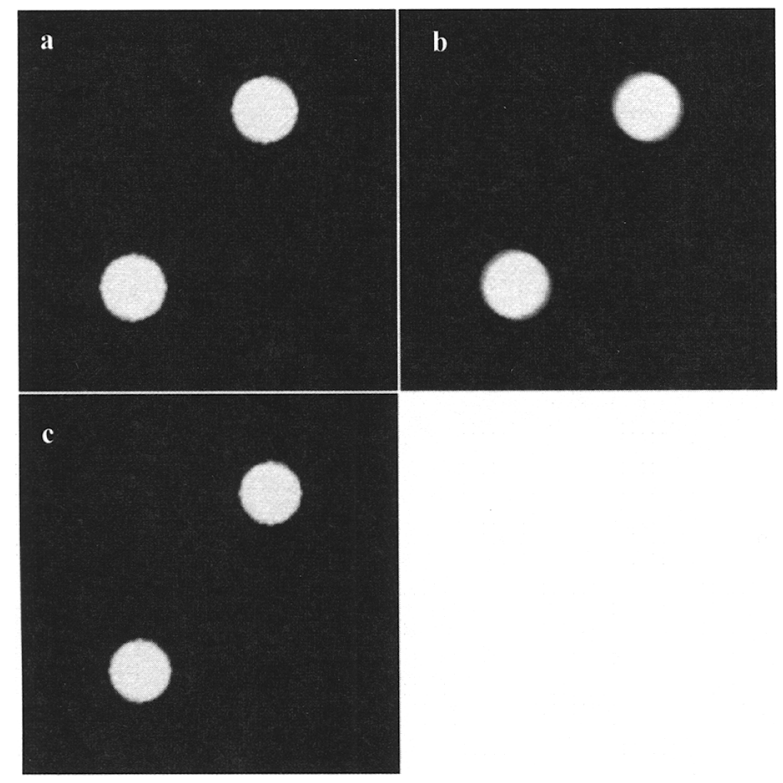

Fig. 6: 6 pixel Y-shift (a) and 1-pixel X-shift (b). Image dimensions: $200 \times 200 \times 100$ pixels. Axial view.

TABLE I

TOLERANCE TO MISADJUSTMENTS

\begin{tabular}{llllll} 
Misadjustment & Roll & Pitch & Skew & X-shift & Y-shift \\
\hline $\begin{array}{l}\text { Minimum value with } \\
\text { visible effect }\end{array}$ & $\sim 8^{\circ}$ & $\sim 8^{\circ}$ & $\sim 0.5^{\circ}$ & $<1 \mathrm{px}$ & - \\
\hline
\end{tabular}

Table I: Misadjustments and corresponding tolerances observed for the phantoms described and a pixel size of $0.25 \mathrm{~mm}$

\section{DISCUSSION AND CONCLUSION}

Skew and X-shifts were the most noticeable misalignments, given that slight errors (about $1^{\circ}$ or sub-pixel shifts) produced conspicuous double edges in the image.

Both types of tilt elongated the image, due to points farther from the central plane suffering a greater deformation. However, this effect proved negligible for usual residual angles of misalignment after calibration (under $3^{\circ}$ ). Y-shifts produced no visible effects either.

Calibration is unlikely to completely remove skew and $\mathrm{x}$ shift misalignments, so a software correction of the projections before reconstruction will be necessary.

\section{REFERENCES}

[1] Y.-X. Wang et al., "Effects on spatial resolution of CT image caused by inaccurate angle localization,", Proceedings of 2005 International Conference on Machine Learning and Cybernetics

[2] M. Karolczak et al., "Practical Approach to Misalignment Correction in a Single-Circle Orbit Cone-Beam Tomography," unknown, 2001.

[3] Y. Sun, Y. Hou and J. Hu, "Reduction of Artifacts Induced by Misaligned Geometry in Cone-Beam CT," Biomedical Engineering, IEEE Transactions on, vol. 54, pp. 1461-1471, 2007.

[4] E. Lage et al., "Design and Development of a High Performance MicroCT System for Small-Animal Imaging," 2006 Nuclear Science Symposium and Medical Imaging Conference (IEEE), pp. 79, 2006. 